\title{
Design and implementation of digital fire control system based on BIM
}

\section{and 3DGIS}

\author{
Tianping $\mathrm{Bi}^{1, \mathrm{a}}$, Peiwen Wang ${ }^{2, \mathrm{~b}}$, Qiang Zhang ${ }^{3, \mathrm{c}}$ \\ ${ }^{1,2,3}$ Shenyang Jianzhu university,school of management,China \\ a57036475@qq.com, b919800456@qq.com, '965892317@qq.com
}

\section{Keywords: Digital fire,BIM,GIS}

Abstract.The independent smoke detector play a very important role in the prevention and control of fire. Combine BIM technology with GIS technology to improve the level of emergency services and fire control, reduce fire risk, improve the depth of 3D information technology and the ability of fire warning. Firstly, we use Revit software to build building and fire equipment models. Secondly, based on the Skyline development platform, use C\# with .NET as development language and Oracle as the database. Finally, use Revit AP to develop the Revit model, complete the transformation interface with 3DGIS. In this way, the BIM model can be converted into 3DGIS and urban digital fire control system. The fire protection remote monitoring system is connected to the Internet. The fire control points have the perfect early warning mechanism to avoid the misstatement caused by waste, which can be used to ensure the safety of people's life and property. The design and implementation of digital fire control system based on BIM and 3DGIS is helpful for the technical support of on-site rescue and post-disaster assessment. It also provides reference for the construction and research of relevant intelligent fire fighting platforms.

\section{Introduction}

The application of building information model is widely applied, and BIM technology can be integrated into the structure of building fire fighting, which can improve fire control management level ${ }^{[1]}$. The combination of BIM and GIS technology creates a comprehensive application platform for information matching, which can give play to the advantages of data information technology in the fire control of high-rise buildings ${ }^{[2]}$. The core of digital fire fighting is the support and supervision of data. Reduce fire risk by playing a preventive mechanism. Rely on management level to guarantee the safety of people's life and property.

GIS technology has been applied early in the current domestic digital fire fighting. Wen Zha (2010) proposed to use GIS as the main module of fire communication function to realize the query of the fire protection geographic information system ${ }^{[3]}$; The application prospect of GIS in fire was analyzed from four aspects: fire risk assessment and monitoring, site selection of urban public fire protection facilities, disaster prediction and auxiliary decision simulation ${ }^{[4]}$. The development of digital fire fighting technology in foreign countries mainly includes military and commercial aspects. The use of digital radio communication is proposed in the field of civil to shows fire alarm in signal ${ }^{[12]}$. To sum up, the concept of digital fire control and applied value though proposed earlier but lack of practicality. It does not realize the 3D visualization of digital fire control and the use of scientific management to reduce risks. Based on BIM and 3DGIS in the actual project, the author has developed a digital fire fighting system with practical significance. The paper introduces the value, design principle and operation method of the system in the field of fire fighting. 


\section{Digital fire fighting system architecture}

\section{System design thinking}

The skyline 6.6 platform and .NET Framework 4.0 are the basis of this system, and C\# is the programming language. Use Oracle $11 \mathrm{~g}$ database for data management and analysis, the 3D scene simulation and spatial analysis of image data, DEM data, building model data and fire resource data are realized. The overall design of the system applies the three-tier architecture of $\mathrm{C} / \mathrm{S}$ mode. The presentation layer, the business layer, and the data access layer are separated. The business layer includes the application layer and the component layer.

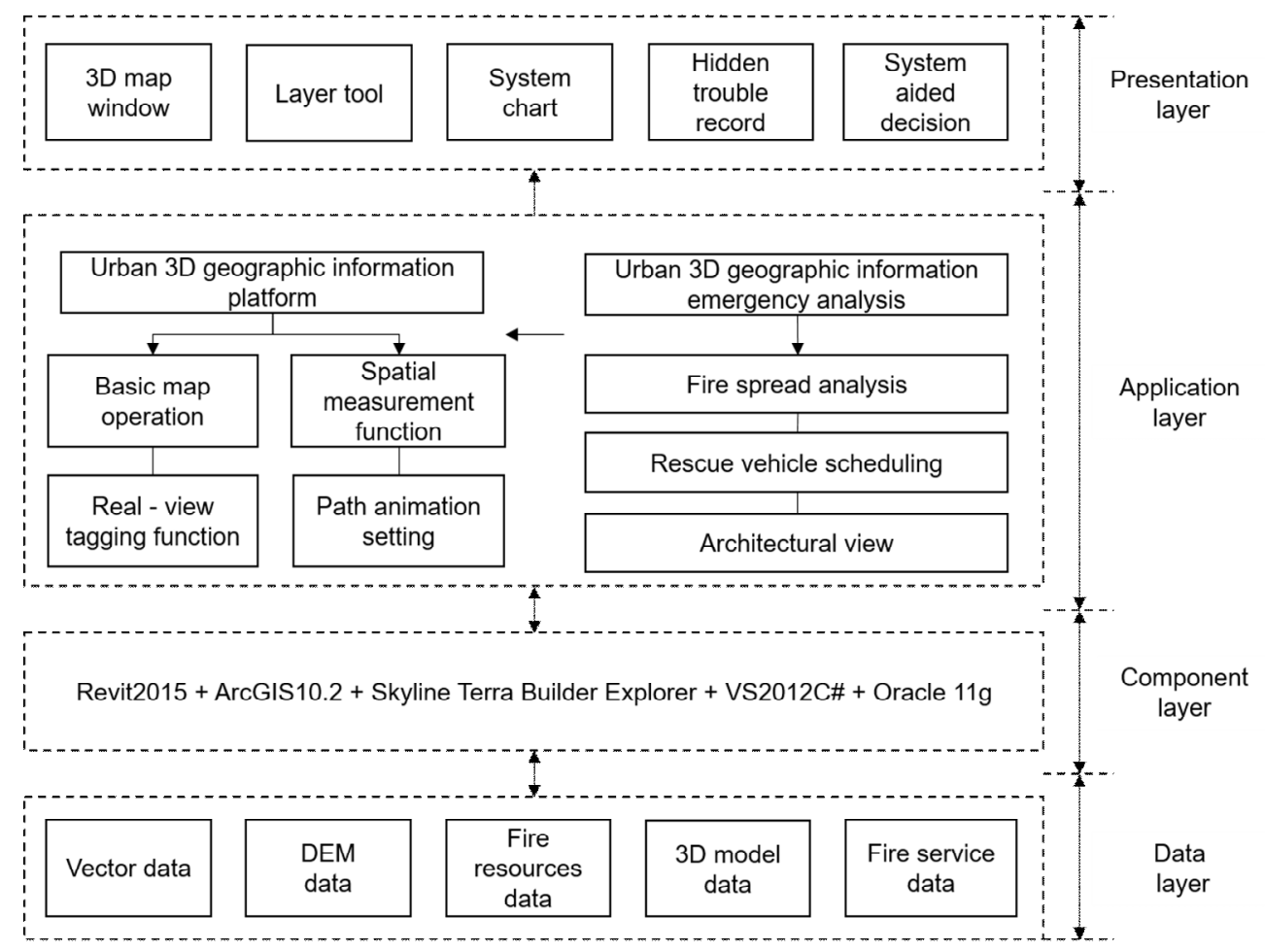

Fig. 1 system structure diagram

The presentation layer includes main window, function window and other Windows, mainly provide interactive interface for users. The main window includes the system information tree, navigation graph, toolbar and 3D analysis and query result window. The function window is used to display fire emergency module and input relevant data and parameters. The application layer is the core functional area of the three-dimensional fire protection system, which is realized by the expansion of fire service business, the model component library and the application layer. The data layer mainly includes vector data, DEM data, 3D model data, fire resources data, etc.

\section{Implementation of digital fire fighting system}

Combine the technical integration of BIM technology and GIS technology is the key to realize digital fire prevention. Specific technical operation procedures are as follows:

Firstly,Use Oracle software as the support for data integration and storage, and build an Oracle server;Then, Use the C\# language development, Visual Studio 2012 software was used to complete the development of the main interface of the TerraExplorer software in Skyline6.6.1, and the function modules of login, query, classification, position and attribute analysis were completed. Thirdly, We redeveloped Revit through Visual Studio 2012. Install the plugin in the software and add 
the "export .USX" module in the attachment module;Lastly,Data interaction and hosting is our goal. First, export the Revit model. Next, convert the .USX format to .X format. Finally, 3D Explore is used to import model data into TerraExplorer software.

\section{The role of BIM in this digital fire control system}

BIM technology can play an important role in fire. It can realize two-dimensional and three-dimensional model construction according to the drawing.In the digital fire control system, fire fighting equipment, drainage pipes, water tanks, fire smoke detectors, fire control temperature detectors and control modules can be displayed in 3D.After that, use the skyline software and the building model was placed in a three-dimensional space model . The advantages of BIM technology can be reflected in the following aspects:

(1)To facilitate the maintenance of fire equipment. The fire equipment has "exposed" and "inner hidden" categories in the building. BIM technology shows the fire equipment in a three-dimensional visual way to facilitate the control of pipeline layout and equipment location. Fire equipment specifications and model information can be entered into BIM system. Therefore, the operation frequency can be reduced in the equipment tracking, and the security hole can be identified.

(2) BIM technology can be used to determine whether fire prediction is accurate. The fire has an expanding process. Smoke alarm system can detect smoke concentration, and when it exceeds the limit, it will be warned. However, in some cases, smoke alarms will fail, using BIM technology to view the space logic between the alarms to determine if there is a fire. If the alarm device is discontinuous in space, and there is no fire in the alarm location, it can be judged that it is an alarm failure. So, BIM technology can reduce the human and material loss caused by false alarm.

(3) BIM technology can establish safe rescue passageway according to the location of fire situation and fire spreading model. On the one hand, it can be led by professional security personnel to retreat, reduce and avoid loss of life and property; On the other hand, fire rescue personnel use BIM technology to understand the structure of buildings and the location of fire-extinguishing equipment so as to facilitate the rescue of the life and property of the masses.

\section{The role of GIS in this digital fire protection system}

Since the BIM model don't has a coordinate concept, it is necessary to make up for it by GIS technology. The visualization of GIS technology shows the unit coordinates of the graph. According to the precision coordinate, it can reduce the identification error, improve work efficiency and save time. GIS technology at present stage more than simply used for fire control facilities location tagging. In order to give full play to the role of GIS technology, 3D positioning digital fire protection construction is adopted in this project.The geographical information system database is improved by collecting the geographical space image and vector map of the project location. Finally, the 3DGIS is modeled through the TerraExplorer 3D digital earth platform.

In this digital fire fighting project, the main functions of GIS technology are as follows:

(1) GIS technology is used to collect and establish evaluation system to realize fire risk assessment. GIS technology can integrate information such as climatic conditions, architectural structure, equipment status, traffic condition, path simulation, etc., and put information in the GIS database. First, the superposition analysis and diffusion analysis are carried out. Secondly, analyze the indicators and weights of each part and establish the evaluation system. Finally, use GIS to develop rescue plan and complete the division of fire area. 
(2) The function of ArcGIS software model is used to realize automatic fire risk assessment. In order to avoid the major loss caused by fire, we adopt the method of numerical calculation of space. This method can estimate the risk range after fire by simulation of spatial risk parameters. Through stacking analysis and buffer analysis, fire spreading model is generated. In addition, GIS technology and GPS technology are combined to realize the monitoring of fire control points.

(3) Rely on GIS electronic map to optimize rescue plan. The planning of fire monitoring should take all factors into account to ensure the accuracy of the results. Firstly, use GIS electronic map, the location information of firefighting equipment can be provided to the user. This can improve the efficiency of firefighters. Secondly, transportation routes can be planned. Ensure that fire crews and medical teams can reach the scene in the shortest possible time.

\section{Conclusion}

Building an adaptive digital fire system is key. It should fundamentally solve the problems such as prevention, rescue and accident analysis of fire accident. It also fully embodies the modernization and informationization of fire fighting. At present, the technology of the project is advanced and it is expected to provide technical solutions for future smart city construction. It meets the efficient and convenient application demand of information era. It not only provides technical ideas for the digital fire protection system of B/S architecture, but also meets the requirements of fire information and 3D functions. The integration of BIM and GIS technology is also important in the realization of 3D function in other industries. Based on current functional testing, our research and development will shorten the modeling period. Whether it is cost saving or functional configuration, the system has a certain degree of advantage.

\section{Acknowledgements}

This work was financially supported by 13th Five-Year National Key Research and Development Programs (2016YFC0702002), Liaoning Provincial Natural Science Foundation (20170540759; 201602604), Liaoning Provincial Department of Education general project (LJZ2016005) and Shenyang Jianzhu University general project (2017101).

\section{References}

[1] Rong Ren. Building fire protection system optimization based on BIM technology [D]. Zhengzhou university, 2015.

[2] Lin Wang,Dongmei Huangpu,Zhen Jia. The influence of BIM technology on fire command and rescue in high-rise buildings [J]. Construction technology, 2015,(18):49-52.

[3] Wen Zha. The application of GIS technology in fire control communication command system [J]. Technology information, 2010,(03):454.

[4] Kun Du,Dong Wei. The application of GIS in the field of fire protection [J]. China public safety (academic edition), 2015,(01):70-74.

[5] Liping Zhu,Rongfang Shen. Digital fire control -- the inevitable trend of the modernization of fire control management $[\mathrm{J}]$. Journal of tongji university (social science edition), 2003,(03):98-102. 